LBL -32810

DE93 002568

\title{
MICROMECHANICS OF SHEAR BANDING
}

John J. Gilman

Operations Division

Lawrence Berkeley Laboratory

University of California

Berkeley, CA 94720

August 1992

This work was supported by the U.S. Department of Energy under Contract No. DE-AC03-76SF00098. 


\title{
MICROMECHANICS OF SHEAR BANDING
}

\author{
John J. Gilman \\ Lawrence Berkeley Laboratory \\ Berkeley, California \\ 94720
}

\begin{abstract}
Shear-banding is one of many instabilities observed during the plastic flow of solids. It is a consequence of the dislocation mechanism which makes plastic flow fundamentally inhomogeneous, and is exacerbated by local adiabatic heating. Dislocation lines tend to be clustered on sets of neighboring glide planes because they are heterogeneously generated; especially through the Koehler multiple-cross-glide mechanism. Factors that influence their mobilities also play a role. Strain-hardening decreases the mobilities within shear bands thereby tending to spread (delocalize) them. Strain-softening has the inverse effect. This paper reviews the micro-mechanisms of these phenomena. It will be shown that heat production is also a consequence of the heterogeneous nature of the microscopic flow; and that dislocation dipoles play an important role. They are often not directly observable, but their presence may be inferred from changes in thermal conductivity. It is argued that after deformation at low temperatures dipoles are distributed a 7 a Pareto so there are many more small than large ones. The instability at the upper yield point, the shapes of shear-band fronts, and the mechanism of heat generation are also considered. It is shown that strain-rate acceleration plays a more important role than strain-rate itself in adiabatic instability.
\end{abstract}

\section{INTRODUCTION}

The idea that heterogeneities play a critical role in the strengths of materials has a long history. Attempts to quantify this idea go back at least 100 years to J. Larmor (1892). Larmor made the point that stresses are concentrated at flaws and cavities (especially elliptical ones) so applied stresses become internally magnified. He did not explain, however, why his arbitrarily large internal stresses did not cause all solids to fail under small applied stresses. That remained for A. A. Griffith (1920) to explain. The extension to plastic solids by $E$. Orowan (1934) completed the rationalization for tensile stresses. 
A crisis arose regarding shear strength. X-ray diffraction had clearly established the atomic structures of solids, and a satisfactory theory of cohesion had been developed by 0 . Madelung (1910), and by the school of M. Born (1933). It was known that salt crystals ( $\mathrm{NaCl}$ ) are often quite soft (yield stress about $1 \mathrm{MPa}$ ), but a simple theoretical estimate by J. Frenkel (1926) based on homogeneous deformation gave a yield stress of about G/15 $\approx 10^{3} \mathrm{MPa}$, where G is the shear modulus. All attempts to reconcile the thousandfold discrepancy on the basis of homogeneous processes failed, so it was concluded that a heterogeneous microscopic process must be operative. A satisfactory process was proposed almost simultaneously by Orowan, Polanyi, and Taylor in 1934 (Cottrell, 1953). This was, of course, the motion of dislocation lines. At first dislocations were considered principally in two dimensions. It was only after researchers began to think of them in three-dimensional contexts that satisfactory models of multiplication and strain-hardening could be developed.

It has been known for a long time that most of the work that is done on a material during plastic deformation is converted into heat in most circumstances ( $90-95 \%$ of $i t)$. The actual conversion mechanism has not been clearly identified. Probably because there is more than one. It is known, however, that it is not a consequence of linear interactions, but appears to be related to nonlinear phenomena such as: strong dislocation-core/crystal interactions; "plucking" of dislocation lines; and annihilation processes.

If the local plastic shear-rate is sufficiently high, the heating-rate will exceed the local cooling-rate, leading to adiabatic instability as the plastic resistance of the material decreases. This was dramatically demonstrated by Zener and Hollomon (1944) for the punching of steel during World War II. They found that the shear zone during fast punching becomes hot enough to locally austenitize the steel, and when the surrounding steel subsequently quenches the shear zone, it becomes martensitic. An historical note regarding this dramatic experiment has been kindly provided, for this symposium, by Dr. Clarence Zener: 
"I am happy for this opportunity of sharing with you my memory of my close association with John Hollomon which led us to recognize that adiabatic deformation can induce catastrophic slip.

The time was January 1942; the location was Watertown Arsenal, near Boston. This arsenal was responsible for the design of armor plate to resist penetration, and of projectiles to penetrate armor. I had just published a paper which gave a perfect correlation between theory and observation on the elastic rebound of steel balls from plates. The ordnance department thought I might help in their design problems. They had contacted MIT for metallurgical advice. MIT recommended John Hollomon, who had just received his doctorate in metallurgy, together with an advancement to a lieutenancy in the Army.

When John and I first met at the Arsenal that January, we recognized the need for close collaboration. We agreed that he would teach me metallurgy, and I would teach him physics. The concept of cataclysmic slip resulting from an instability in adiabatic shear deformation arose from this collaboration. Speaking of collaboration reminds me of the tremendous help both John and $I$ received from the Commanding Officer of the Arsenal, Colonel Zornig. He fought a battle with his superiors in Washington to allow us to publish our joint work, which finally resulted in seven papers on physical metallurgy. While admitting that publication would help the Germans, Colonel Zornig argued that classifying the results would prevent their wide use in this country. Conventional wisdom speaks of the flow of ideas from pure research to applied research. The war years alerted me to the counter flow. I have never been so productive in theoretical concepts as during the war years when I was continually bombarded with practical problems." 


\section{PLASTIC FLOW}

Plasticity is a transport phenomenon like those in which heat, charge, or mass, are transported. In the case of plasticity it is displacement (shape) that is transported. In all four cases (plasticity, thermal conduction, electrical conduction, and diffusion) the form of the equation that connects the macroscopic observable flux with the microscopic events is the same. The macroscopic flux is given by the product of: a characteristic quantity, the concentration of the quantity, and the average transport velocity. For plasticity this is known as the Orowan equation. One way in which plasticity differs from the other transport phenomena is that the underlying atomic events that cause it do not occur at random throughout a volume, but are highly correlated within at least three, and perhaps four levels of aggregation. This distinguishes it from its close relative; Newtonian viscosity.

Since the macroscopic strain-rate is proportional to the product of the concentration of carriers (dislocations), and their average velocities, a macroscopic measurement always has an ambiguous microscopic interpretation. For a given value of the product, the concentration may be high while the velocity is low, or vice versa. Also, it provides various irreversible paths to reach a particular plastic state, so no equation-of-state exists.

Shear-banding is a consequence of the correlation of microscopic plastic events. At the atomic (or molecular) level, the most elementary shear events occur at kinks along dislocation lines. Thus they are not independent as in a simple liquid, but are correlated. The kinks on the dislocation lines occur in pairs with opposite signs (at a surface one member of a pair is virtual). Each kink carries a characteristic displacement, $b$. The average velocity, $v_{k}$ of the kinks, and their concentration, $c_{k}$ determines how fast their associated dislocation line moves. Its velocity, $v_{d}$ is given by:

$$
v_{d}=b c_{k} v_{k}
$$

when $c_{k}$ becomes saturated at $-1 / b$ the two velocities become approximately equal. 
SHEAR-BANDING VIA MULTIPLE-CROSS-GLIDE

Although many papers were written in the 1940-60 period expressing the idea that shear-bands (as implied by upper yieid points) resulted from the release of pinned dislocations from Cottrell impurity atmospheres, this idea was unsatisfactory because dislocation motion is highly dissipative (viscous). Therefore the average value of the velocity always corresponds to the instantaneous value of the stress. That is, dislocations do not "run away"; their velocities are steady at constant stress. Perhaps the first person to clearly recognize this was Holden (1951) who proposed a collision cascade mechanism for yielding: similar to the electron avalanches of dielectric breakdown theory.

The steadiness of average dislocation velocities was demonstrated by observing the distances traveled by dislocations acted upon by stress pulses of unit length, as well the distances travelled when pulses twice as long were used. In the latter case the distances were exactly twice as long (Gilman and Johnston, 1962). This proved that the motion is steady. The intrinsic reason for the steadiness in orthotropic crystals is the multiple-cross-gliding that will be discussed shortly. This produces drag that is much greater than the underlying drag at the atomic lever.

The existence of avalanches can be deduced quite simply from the kinematics of dislocation behavior. That is, from the Orowan transport equation together with the observation that the dislocation density increases lineariy with the plastic strain at constant stress (constant velocity). From the orowan equation, an increment of plastic strain during a time interval, dt is given by:

$$
d \epsilon_{p}=(b N v) d t
$$

where $b=$ Burger's displacement, $N=$ dislocation density $\left(\# / \mathrm{cm}^{2}\right)$, and $v=$ average dislocation velocity. Neglecting the small initial dislocation density:

$$
N=\alpha \epsilon_{p}
$$

where $\alpha$ is the proportionality constant. Then, letting $A=$ constant factors $=$ bva, and using Eq. (1): 


$$
d N / d t=A N
$$

showing that first order kinetics is acting. Upon integration:

$$
N=N_{0} \exp (A t)
$$

which describes the exponential growth of an avalanche.

The process that yields the first order kinetics of Eqn. (3) was first clearly described by Koehler (1952); and was experimentally confirmed in detail by Johnston and Gilman (1960). It comes from the symmetry of screw dislocations. In an elastically isotropic medium, screw dislocations are cylindrically symmetric except at their cores. Therefore, they can move in any direction normal to their line tangent with nearly equal ease. The plane of maximum shear stress will be favored, but there are always two of these. Also, the crystallographic symmetry around the Burgers vector, and the extension of the core (or lack of it) may bias the direction of motion. The net result is that there is often little to restrain them from wandering from one glide $p l$ ane to another and then back to a plane parallel to the original one as illustrated in Figure 2. The way in which internal stresses cause the multiple-cross-gliding is described in the theory of $\mathrm{Li}$ (1961).

The motion of a single dislocation half-loop creates an elementary shear event (Figure 1). This corresponds to a macroscopic strain of $\sim 3 \times 10^{-13}$. However, through multiple-cross-glide (Figure 2) a dislocation line can undergo "random walk" onto a parallel neighboring plane. This creates two jogs: $\alpha \beta$ and $\delta \gamma$. They lie perpendicular to the Burgers vector, so they can move parallel, but not perpendicular to it. If the distance, $H$, between the first primary glideplane and the second one is large enough, the lines on the top and bottom $p$ lanes can pass over one another to create the configuration of Figure 3, followed by that of Figure 4. In this way, the original screw dislocation of Figure 2 can undergo "mitosis" creating two lines from one.

If the distance is too small for the applied stress to push the dislocations on the top and bottom planes over one another, the configuration becomes 
like Figure 5. That is, the two jogs remain connected by edge dipoles to the original dislocation as it moves forward. This produces drag on the moving dislocation which is the beginning of strain-hardening, and is the basis of the Bauschinger effect.

The dipoles from the non-crossover case can only be eliminated by climb. This is diffusion controlled, so at low temperatures they accumulate and impede the motion of other dislocations thereby causing strain-hardening.

As the screw dislocations continue to cross-glide, a microscopic shear-band is created; and eventually a macroscopic one. The dipoles that are left behind strain-harden the centers of shear-bands, but the bands remain active at their two sides until they have widened so much that they impinge on each other. Then the material will have become strain-hardened everywhere.

When plastic shear-bands become macroscopic they are called Lueders bands (steel), or draw-necks (polymers). In individual crystals they are called glidebands, or slip-bands.

In principle, a highly deformed macroscopic crystal may contain just one dislocation line (including its virtual connectors at surfaces). This one line will have been derived from just one initial critical nucleus. The length of such a line in a one centimeter cube of material might be as much as $10^{8}-10^{9} \mathrm{~km}$; or enough to stretch from the Earth to the Sun.

The final level of aggregation is that of a polycrystalline material; or one grain therein. Since, as will be explained, the underlying mechanisms allow for the existerice of negative plastic resistance (do/de $<1$ ), polycrystalline aggregates may have the same. This leads, of course. to macroscopic shearbanding.

There are other modes of plastic instability in solids. For example, macro-kinking due to shear $\mathrm{plane}$ rotation, micro-kinking due to the formation of dislocation dipole arrays, twinning, and molecular ordering in polymers. However, the discussion here will be limited to the effects of dislocation 
multiplication, cohesion degradation, and heat production.

The experimental evidence obtained by Johnston and Gilman (1960) is quite clear. By means of surface etching and chemical polishing techniques, they showed that single half-loops of dislocation line when expanded by applied stresses can become shear bands containing innumerable dislocation loops, and dislocation dipoles (Figure 6 ). This figure shows six pairs of surface etch-pits representing a set of six dislocation half-loops; then it shows the surface offsets that appeared after shear stress was applied to them; and finally the substructures of multitudinous loops within the shear bands.

Such bands grow by expanding laterally (Figure 7). As long as they can do this without interfering with one another there is little macroscopic strainhardening, but when a specimen becomes filled with the initially operative shear bands, the macroscopic stress begins to rise (Stokes, 1964) as illustrated by Figure 8.

Another general feature of the multiplication process is that the rate is very stress (or velocity) dependent (Gilman and Johnston, 1962). Thus it was shown that a half-loop can be expanded slowly (under a small applied stress) without causing it to multiply, but if it is expanded quickly (under a higher stress) it will multiply profusely. This is one of the fundamental reasons why there is no plastic equation-of-state in terms of the macroscopic variables. Figure 9 shows the data of Gilman and Johnston plotted in a different way in order bring out the strong stress dependence of the multiplication process. It shows the variation of the logarithm of the linear dislocation loop density with the reciprocal shear stress. It may be seen that changing the stress by a factor of two leads to a change in the multiplication rate by as much as three orders of magnitude.

\section{LOCALIZATION}

In order to understand how adiabatically unstable shear bands form, it is 
important to recognize that they do not represent thermal instability alone. Geumetric instability is also involved through the multiple-cross-glide mechanism because this process can continue to generate dislocation lines after there is already a sufficiency of line length to provide a macroscopic displacement-rate equal to the applied displacement-rate. When an excess is produced in a specimen that is mounted in a stiff machine that applies a constant displacement-rate, the applied load drops and yielding is said to have occurred.

If, in addition to the excess dislocation concentration generated by multiplication, local heating increases the average mobilities of the dislocations, the displacement-rate of the specimen further exceeds that of the loading machine so the load drop becomes larger. The most apparent way for the mobility to increase is for dipoles to be annealed out, thereby removing strainhardening (Gilman, 1962).

The resistance to thermal conduction in metals can be expected to increase significantly within shear bands as the defect concentration in them increases (Berman, 1976). The conductivity will decrease due to defect scattering of electrons which are the major carriers of heat in metals. In insulators where phonons are the major heat carriers, there will be little change except at temperatures well below the Debye temperature of the material. The major defects that are produced are dipoles so we need to consider how they form and they distribution in somewhat more detail. As will be shown later, dipole production a) so plays an important role in the mechanism of conversion of plastic work into heat.

In addition to -ausing strain-hardening, dipole trails have various other effects. They scatter phonons, so they decrease thermal conductivity. They also affect specific heat because they can vibrate as harmonic oscillators; introducing "soft" local phonon modes. Also, when they are present at sufficiently high concentrations they degrade the local sirength of the material. They are probable also responsible for the low-temperature internal friction known as 
Bordoni friction.

The crossing-over criterion for dislocations is well-known (Cottrel1, 1953) thereby setting the largest jog size at $G b / 8 \pi(1-v) \tau$ where $G, b$, and $v$ have their usual meanings and $\tau$ is the applied shear stress. The smallest jogs are single glide-plane spacings in size (leaving behind dipoles that are equivalent to rows of either vacancies, or interstitials). Since the cross-gliding process is stochastic, but constrained to the special regions of the material where most of the dislocation motion is (typically between the strain-hardened parts and the virgin parts), dipoles of all allowed sizes form. Therefore, they are distributed according to a Pareto rule (at low temperatures). This distribution is a key to under-standing the behavior of shear bands.

The most frequent jogs (dipole heights) are expected to be the small ones. This is true during the initial formation of the shear bands. Later as the frequency of interactions increases, the distribution will tend to become uniform (all sizes equally probable; although there is such a jumble that the definition of "size" becomes murky). Initially, small jogs are most frequent because the probability of cross-gliding increases with stress, and so does the probability of returning to the primary glide plane.

Standard methods of microscopy do not reveal the small dipoles. First, because they are quite fragile. They are attracted to free surfaces where they become erased. Also, they s:an become annihilated through climb. Second, since electron microscopes depend on the strain fields of dislocations to produce contrast, and the strain fields of dipoles are very localized, small dipoles are not visible in electron micrographs. And very. small ones are not visible because their fields extend less than the resolution distance (remember that the smallest ones are equivalent to rows of vacancies or interstitials). Perhaps the best experimental evidence for the presence of large numbers of small dipoles is thermal conductivity data. It is found that measured conductivities are much smaller (by 2-3 orders of magnitude) than expected from theoretical calculations; 
based on observable dislocation concentrations (Berman, 1976). This discrepancy is removed if there re large concentrations of unobserved dipoles.

The size distribution rule is quite simple. The aipole flux density per $\mathrm{cm}^{2}$ (length per unit volume), L, of a given dipole height, $h$ is inversely proportional to the height between the limits: $h_{0}, L_{0}$ and $h^{*}, L^{*}$. The height, $h_{0}$ cannot be smaller than approximately, b (2-3 is probably a better estimate); and if a shear stress, $\tau$ is present it cannot be 1arger than about $h *=17(G / \tau) b$. Thus, letting $L(h)$ be the number density of dislocation dipoles of height, $h$, and $A$ be a constant of order unity, the distribution rule is:

$$
L(h)=\left(h_{0} / h\right) L_{0} \quad[2 b<h<17(G / \tau) b]
$$

as Figure 10 illustrates. At its dense end near $L=L_{0}$, the dipoles are not easily observed because the strain fields are highly localized. At the upper limit $\left({ }_{L} 0 \times 3 \times 10^{14} / \mathrm{cm}^{2}\right)$ the material is nearly liquid-like, and the structure is unstable. It tends to recrystallize. Also, the dimensions of the structure are too small to be resolved by most microscopes.

Under steady plastic-flow conditions, the average rates of creation and annihilation of dipoles is just sufficient to convert the external plastic work into heat, at whatever rate it is being done. Local rates may vary, of course, depending on whether the material is (or is not) near grain boundaries, or other interfaces. If the rate is higher than a critical value instability may result.

\section{SHAPES OF SHEAR BANDS}

For shear punching configurations there is not much that can be said about the macroscopic shapes of shear bands. However, the "Lueders bands" that appear during simple tensile loading the bands have definite predictable snapes. This provides a means for checking the validity of the micro-mechanism of formation of the bands. If valid, it should be able to predict the shapes.

It has been shown (Gilman, 1965) that the mobile dislocation density in a shear band increases initially as multiplication through multiple-cross-glide 
occurs, and then decays exponentially with further strain as the dipole concentration builds up. The kinematic equation that describes this is:

$$
N_{m}=\left(N_{0}+M \epsilon_{p}\right) \exp \left(-\phi \epsilon_{p}\right)
$$

The term that precedes the exponential describes the increase in the total dislocation density with increasing plastic strain, $\epsilon_{p}$, while the exponential describes the fraction that remains mobile. $N_{0}$ and $M$ are independently measurable, so the only adjustable parameter is $\phi$.

Equation (6) when combined with Eq.(1) yields an equation for the plastic strain-rate as a function of the strain. The integral form of this is a creep equation which can be expressed in terms of the tabulated exponential integral, $\mathrm{Ei}(\mathrm{x})$. Figure 11 shows how well the theory describes an observed creep curve. With different parameters, the same theory describes creep in a variety of materials. For example, aluminum oxide, and steel. It also describes very fast strain-time curves such as the leading edges of plane-wave shocks, and the delay times for the yielding of steel. Furthermore, the large strain expansion of the creep equation yields the familiar logarithmic creep law (Gilman, 1965).

Because of the continuity condition, Hart (1955) has pointed out that at every point of a shear-band steadily propagating with velocity, $V_{b}$ :

0

$$
(\partial \epsilon / d t)=V_{b}(\partial \epsilon / \partial T)
$$

where 1 is distance, and $\epsilon$ is the longitudinal strain. This means that since the creep equation gives the left-hand side of this equation, the band shape be obtained since $\partial \epsilon / \partial \mathrm{l}$ is known at each point. Thus the shape is expected to be sigmoidal analogous with the strain-time curve of Figure 11; and indeed this is what is observed experimentally. As suggested at the beginning of this section, this provides validation of the microscopic view of shear bands that has been out 1 ined.

\section{RECRYSTALLIZATION}

If the local defect energy density in a volume of critical size exceeds a 
the latent heat of melting, the material will recrystallize. The critical size is determined by the energy of the interface between the recrystallized material and its surrounding; or approximately the average energy of grain boundaries in the material. Since we know the magnitudes of the important parameters we can calculate the expected sizes of recrystallized grains. These can then be compared with the observations of Meyers and Pak (1986).

Consider a material in which the excess (over i perfect crystal) energy due to defects has reached a density equal to the latent heat of melting, $H_{m}\left(\mathrm{~J} / \mathrm{m}^{3}\right)$. If a small volume of radius, $r$ recrystallizes, the energy associated with the defects will be released: that is, $H_{m}\left(4 \pi r^{3} / 3\right)$. But a grain boundary will now surround the new crystal. Let, $\gamma_{g}\left(j / m^{2}\right)$ be the average specific grain-boundary energy, so creating it will require $\gamma\left(2 \pi r^{2}\right)$ in energy. A metastabie eqilibrium will exist when the differentials of these two energies are equal. That is, at a critical radius, $r^{\star}$ of:

$$
r^{*}=2 \gamma_{g} / H_{m}
$$

For titanium: $H_{m}=17.5 \mathrm{~kJ} / \mathrm{mol}$. - Smithells Metals Reference Book, 6th Edition, Butterworths, London (1983).

$$
\gamma_{g}=\text { approx. } 500 \mathrm{~mJ} / \mathrm{m}^{2}
$$

so: $r^{*}$ - $10 \AA$ which is small compared with the recrystallized grains (500-3000 A) seen by Meyers and Pak (1986). Thus their grains may well have grown from much smaller ones.

\section{TRANSIENT MECHANICAL RESPONSE}

Stresses are created in materials by displacing their surfaces relative to their interiors. In virtually all cases this is done at a finite rate, so transients are involved. In the elastic regime the transients are elastic waves which conserve energy. But in the plastic regime, the transients cause permanent shape changes which come about through dislocation motion and multiplication. The external work is absorbed as heat, and internal configuration changes. Since the internal changes are microscopically heterogeneous they form through the 
propagation of fronts through the material. These transient fronts may be large (at the upper yield point, for example); or they may be small and manifest themselves as serrations on a stress-strain curve; or they may be very small manifesting themselves as bursts of low level acoustic noise; or they may be very, very small and not normally observable. Since transients are always present in materials with discrete atomic structures, they need to be considered; especially the large ones.

In a broad sense, there are two mechanisms for the production of plastic transients. One is the "avalanche" mechanism, and the other is the "trafficflow" mechanism. The former results from multiplication kinetics, while the latter results from the collective interactions within sets of moving dislocations. These collective interactions resemble those within lines of vehicular traffic (Gilman, 1968). They will not be discussed further here.

In order to understand macroscopic transients, the expressions for the time derivatives of the strain in terms of the microscopic variables must be understood first. There is some confusion in the literature regarding these expressions which appears to derive from the fact that dislocations are 1 ine, not particles; thus they do not have mean-free-paths, for example. Dislocation lines are the edges of surfaces, so they are actual or implied loops. They cannot end inside a homogeneous material.

A simplified approach to the strain derivatives is to use a mean loop approximation. That is, to replace the complex array of loops that exists in real materials by an equivalent concentration, $n$ of mean loops per unit volume. Furthermore, although real loops tend to be elliptical with jogs and kinks along them, let the idealized ones be smooth and circular with radii, $r$. Then they will have perimeters, $p=2 \pi r$, and areas, $a=2 \pi r^{2}=p r / 2$. Let an average area increment be da. Then, a small change in plastic strain, $\epsilon$ will be:

$$
d \epsilon=(b n) d a
$$

where $b$ has the usual meaning. Now take the time derivative: 


$$
\begin{aligned}
d \epsilon / d t & =b(n d a / d t+d n / d t d a) \\
& =(b n) d a / d t
\end{aligned}
$$

since the second term in the parentheses is second order (this is crucial step, true for lines but not points, because as dt becomes infinitesimal, an infinitesimal loop, dn, sweeps out an infinitesimal area).

Now: $d a / d t=2 p d r / d t=2 p v$

so: $d \epsilon / d t=2 b n p v ; \quad$ or: $b L v$, where $L=n p$ is the line length per unit volume.

Thus, at any constant strain-rate, there is no $\mathrm{dn} / \mathrm{dt}$ term. However, if the strain-rate is changing, we have the following:

$$
d^{2} \epsilon / d t^{2}=(d \epsilon / d t)[(1 / v)(d v / d t)+(1 / L)(d L / d t)]
$$

If the velocity is very high, approaching the sound speed, $d v / d t$ becomes very small so the first term drops out, but the second term which describes multiplication remains. It may be written:

$$
d(\operatorname{lnL}) / d t=d(\operatorname{lnn}) / d t+d(\operatorname{lnp}) / d t
$$

where the first term on the right describes the nucleation of new loops (plus any annihilation that occurs); and the second describes the growth of existing ones (plus any shrinkage that occurs). Note that both describe first order kinetics so the total line length grows exponentially in time.

The theory of plastic yielding that was developed using these expressions generates both upper and lower yield-points without adjustable parameters (Gilman and Johnston, 1962). The upper yield-point is determined by a balance between the displacement-rate created by the applied tractions (as modified by the elastic response of the macroscopic loading system), and the displacement-rate response of the specimen. The latter is determined by: the elasticity of the specimen; the average dislocation velocity in the specimen (a known function of the local stress); the local dislocation density (a known function of the local plastic strain); and the dislocation multiplication-rate (a known function of the local stress).

When the applied displacement-rate exceeds the response-rate of the 
specimen, the stress rises, and inversely the stress falls. If the two rates are equal the stress remains constant (at the upper yield-point). To generate a lower yield-point strain-hardening must be added to the theory.

The internal distribution of dislocations is generated inhomogeneously by means of multiple-cross-glide (which is a function of stress and the local dislocation density). It is not a material constant, nor is it primarily generated by thermal fluctuations. Therefore, the state of the material cannot be described in terms of macroscopic variables alone. That is, there is no plastic mechanical equation-of-state.

For small applied displacement-rates, and specimens that contain some dislocations initially, the stress in the specimen rises initially at a nearly elastic rate. Then, as the product of the average dislocation velocity and the dislocation density increases, the specimen's displacement-rate increases and the rate-of-rise of the stress in the specimen decreases until it becomes zero. No upper yield-point is observed.

As the applied displacement-rate increases a transient develops (overshooting). The displacement-rate response of the specimen lags the applied displacement-rate. After the two rates have become equal, the response-rate of the specimen continues to increase (because the dislocations in it continue to multiply). This relaxes the elastic strain in the external system, so the stress in the specimen decreases. As the applied displacement-rate increases further, the maximum stress in the transient increases. In the limit of very high rates, since the average dislocation velocity is limited by the velocity of sound, and this limits the multiplication-rate, the specimen response becomes essentially elastic.

For very high applied displacement-rates (impact) inertial effects plus the response-rates of detectors must be considered, but there is no reason to expect that there will be a qualitative change in the microscopic mechanisms (in spite of the comments of some authors). 
It has sometimes been asserted that dislocation multiplication is not fast enough to yield an adequately fast response of a material subjected to a strong impact. It is easy to show that this is not the case, using Equations (8-10).

The drop in stress at the upper yield point implies localization into shear bands (Hart, 1955). Thus localization can occur under isothermal conditions. Localization is enhanced, of course, is significant adiabatic heating occurs.

\section{HEAT GENERATION}

In the section above we have shown how shear bands can form through microscopic dislocation motions. But the process that has been outlined contains highly correlated events, not the random ones associated with heat. How then does the conversion of plastic work to heat occur?

The first idea that occurs to most people is that the conversion is due to the "rubbing" of one atomic plane over another at the cores of moving dislocations. In other words due to the glide-plane viscosity. In most cases, especially in metals, this effect is too small, however. Put simply, viscosity consists of the transfer of momentum perpendicular to the direction of shear flow so as to slow down the faster material and speed up the slower. The momentum of a particle with temperature $T$ is $\operatorname{SQR}(m k T)$ where $m$ is its mass, and $k$ is the Boltzmann constant. The damping coefficient that is observed for dislocation motion through ultrasonics is about $B=10^{-4}$ poise, and it equals the momentum transferred per unit area, or $S Q R(m k T) / b^{2}$ where $b$ is an atomic dimension. Thus the maximum local temperature rise is:

$$
T=B^{2} b^{4} / m k=\left(10^{-4}\right)^{2}\left(2.7 * 10^{-8}\right)^{4} /\left(26 * 1.7 * 10^{-24}\right)\left(1.4 * 10^{-16}\right)=0.9^{\circ} \mathrm{K}
$$

which is negligible compared with observations. Thus intrinsic gliding viscosity is not the conversion mechanism in ductile metals. Other approaches to this "rubbing" mechanism are summarized by Nabarro (1987). They reach similar conclusions.

The situation is different when the intrinsic dislocation mobility is low; 
as it is in hard covalently-bonded materials, or in highly disordered metallic glasses. In silicon, for example, damping constants as high as $3^{\star} 10^{5}$ poise have been measured, corresponding to a very high local temperature. However, the motion is slow so the heat is conducted away quickly making the average temperature rise small. Another estimate can be made by equating the energy needed to break a bond in silicon with the thermal energy released; this gives a peak local temperature of about $2.5^{*} 10^{4}{ }^{\circ} \mathrm{K}$.; but again this is quickly quenched in a few picoseconds.

Variants of the rubbing mechanism have been discussed by Coffey and Armstrong (1981), but they do not completely resolve the quantitative deficiencies..

Perhaps the next mechanism that comes to mind is "plucking". That is, a dislocation line is held back locally so it becomes stretched as the rest of it moves forward. Then it is released and it snaps forward, cvershoots its equilibrium position, moves back, overshoots in the return direction. In other words in vibrates about its equilibrium position, generating and scattering phonons (Nabarro, 1937). But the damping constant is small, so it takes many cycles to generate much heat. When the dislocation is released from its pinned position, the "pin" is also set into vibration and may locally reach a high temperature if the force at the time of release is large. But this temperature spike is very local so it cannot contribute much to the average temperature rise. Overal1, plucking does not appear to be a very efficient mechanism for conversion of the input work into heat. Thus it can only be important if the concentration of "plucking points" is high.

A more efficient mechanism of conversion is dislocation annihilation; either partial or complete. Two dislocations of opposite signs lying on the same glide plane that come together will annihilate. Their core energies, elastic strain-energy fields, and kinetic-energy fields will all be converted into phonons with a variety of wavelengths. In units of $\mathrm{Gb}^{2}$ per unit length, these energies are approximately: 


$$
\begin{aligned}
& \text { elastic field }-1 / \pi=0.32 \\
& \text { kinetic field }-1 / 3 \pi=0.11 \text { (velocity = sound velocity } / 3 \text { ) } \\
& \text { core energy }-1 / 8 \pi=0.04 \\
& \hline \text { Total }
\end{aligned}
$$

For iron, if: $G=10^{12} \mathrm{~d} / \mathrm{cm}^{2}$, and $b=2.5 \AA$; the energy released could be as much as $25 \mathrm{e.V}$. per atom plane, or $650^{\circ} \mathrm{K}$ in cylinder of radius $37 \AA^{2}$ (contains about 600 atoms per atom plane). The corresponding flow stress would be about $1.2 \mathrm{GPa}$. which is reasonable for cold-worked iron.

Annihilation processes can be quite varied. Dislocations can emerge from free surfaces; or submerge into grain boundaries; or other interfaces. Those of the same sign can lose most of their elastic and kinetic energies by forming incipient sub-grain boundaries. Those of opposite signs can form dipoles, or be trapped by dipoles so part of their self-energy is eliminated. Those lying on non-parallel planes can meet and react to form new dislocations exothermically. The gradient fields of dipoles can cause attractions between them leading to quadrupole formation, or dipole annihilation. These processes are largely irreversible so they emit phonons and cause heating.

When monopoles, or dipoles approach prior to annihilating one another, they exert large attractive forces which cause them to accelerate to high velocities just before they disappear. This is necessary, of course, in order to produce the large atomic vibration frequencies associated with the high local temperatures that are produced. It would be interesting to know how the conversion ratio of work to heat varies with the macroscopic strain-rate. Can isothermal plastic deformation be approached if the induced strain-rate is very low?

Armstrong, Coffey, and Elban (1982) pointed out that if shear bands are blocked so that dislocation pileups form, and, if the blockage suddenly breaks down, then a burst of annihilation can occur with an accompanying burst of local heating. Their model considers only single-ended pileups, but these seem unlikely because there is a large amount of rotation at the concentrated end of 
a pileup, but the average rotation that is observed in specimens is not large. Therefore, it seems likely that pileups must annihilate in pairs; that is, as "super dipoles". Thus, in deformed monocrystals the initial structure is dominated by a distribution of individual dipoles, whereas in polycrystals the structure contains a large number of super-dipoles in addition to the individual dipoles.

As the dipole density increases in a material, both phonon and electron mean free paths decrease, causing thermal conductivity to decrease; and tending to increase adiabatic instability. The effect is not small. For example, in insulators at low temperatures the change can be a factor of five, or more (Berman, 1976). In metals, changes of a factor of two or more can be expected in highly cold-worked specimens (Nabarro, 1987).

High stress levels concentrate bands of dislocations (and hence dipoles). Thus: low temperatures; high deformation-rates: previous cold-work: and alloying; all tend to localize plastic flow, and lead to adiabatic instability.

The fact that heat generation in metals comes about primarily through annihilation processes has an important general consequence. Namely, that attention must be focussed on Equation (10), rather than Equation (8). That is, the mechanical response that is important to adiabatic instability is the strainrate acceleration; not the strain-rate itself. 


\section{REFERENCES}

R. W. Armstrong, C. S. Coffey, and W. L. Elban, Adiabatic Heating at a Dislocation Pileup Avalanche, Acta Metal1., 30, 2111 (1982).

R. Berman, Thermal Conduction in Solids, Oxford University Press (1976).

M. Born and M. Goeppert-Mayer, Dynamische Gittertheorie der Kristalle, Handbuch d. Phys, XXIV/2, 623, Springer-Verlag, Berlin (1933).

C. S. Coffey and R. W. Armstrong, Description of "Hot Spots" Associated with Localized Shear Zones in Impact Tests, in Shock Waves and High-Strain-Rate Phenomena in Metals, p.313, Plenum Press, New York (1981).

A. H. Cottrell, Dislocations and Plastic Flow in Crystals, p.7, p.159, 0xford Press (1953).

J. Frenkel, Theory of the Elasticity Limits and Rigidity of Crystalline Bodies, Zeit. F. Phys., 37, 572 (1926).

J. J. Gilman, Debris Mechanism of Strain-Hardening, J. Appl. Phys., 33, 2703 (1962).

J. J. Gilman, Microdynamics of Plastic Flow at Constant Stress, J. Appl. Phys., $\underline{36}, 2772(1965)$.

J. J. Gilman, The Plastic Response of Solids, Dislocation Dynamics, Ed. by Rosenfield, Hahn, Bement, and Jaffee, p.3, McGraw-Hill Book Company, New York (1968).

J. J. Gilman and W. G. Johnston, Dislocations in Lithium Fluoride Crystals, in Solid State Physics, Vol. 13, 147 (1962).

A. A. Griffith, The Phenomena of Rupture and Flow in Solids, Phil. Trans. Roy. Soc., 221A, 163 (1920).

E. W. Hart, A Uniaxial Strain Model for a Lueders Band, Acta Meta11., $\underline{3} 146$ (1955) .

A. N. Holden, The Yielding Behavior of Iron Single Crystals, J. App1. Phys., 22, $1290(1951)$. 
W. G. Johrston and J. J. Gilman, Dislocation Multiplication in Lithiun Fluoride Crystals, J. Appl. Phys., 31, 632 (1960).

J. S, Koehler, The Nature of Work-Hardening, Phys. Rev., 86, 52 (1952).

J. Larmor, The Influence of Flaws and Air Cavities on the Strength of Materials, Phi1. Mag., $\underline{33}, 70$ (1892).

J. C. M. Li, Cross Slip and Cross Climb of Dislocations Induced by a Locked Dislocation, J. Appl. Phys., 32, 593 (1961).

E. Madelung, Molekulare Eigenschwingen, Physik. Zeit., 11, 898 (1910).

M. A. Meyers and H. Pak, Observation of an Adiabatic Shear Band in Titanium by High-Voltage Transmission Electron Microscopy, Acta Metal1., 34, 2493 (1986).

F. R. N. Nabarro, Theory of Crystal Dislocations, p.746ff, Dover Publications, New York (1987).

J. F. Nicholas, The Dissipation of Energy During Plastic Deformation, Acta Meta11., I, 544 (1959).

E. Orowan, Proceedings of International Conference on Physics, Vol. 2, p.81, The Physical Society, London (1934).

R. J. Stokes, General Discussion, p.182 in Dislocations in Solids, Discussion Far. Soc. \#38, The Faraday Society, London (1964).

M. H. Tresca, On Further Applications of the Flow of Solids, Proc. Inst. Mech. Engrs., 30, 301 (1878).

C. Zener and J. H. Hollomon, Effect of Strain Rate Upon Plastic Flow of Steel, J. Appl. Phys., 15, 22 (1944). 


\section{FIGURE CAPTIONS}

Figure 1 - Dislocation half-loop enters a surface at the very beginning of the creation of a shear-band. An elementary surface step is left behind. The legs of the half-loop that lie parallel to the Burgers displacement vector are left-and right-handed screw dislocations, and the perpendicular part is of edge-type.

Figure 2 - Because of its cylindrical symmetry, a screw dislocation is not constrained to move on its original glide-plane, but can move off the initial plane onto a cross-glide plane, and then back onto a plane that lies paraliel to the initial one. This is called multiple-crossglide. Note that the jogs, $\alpha \beta$ and $\gamma \delta$, lie perpendicular to the Burgers vector, and are therefore edge dislocations which are constrained to glide only on their current glide-planes.

Figure 3 - As the dislocation lines of Figure 2 continue to move the segment on the upper plane it becomes a semicircle and then a heart-shaped configuration until the two lobes of the "heart" meet, and part of the pair of impinging lines becomes annihilated. At the same time, on the bottom plane the dislocation lines swing forward and laterally until a portion of them becomes annihilated. Note that the upper and lower lines must be able to pass over one another in order for the indicated motions to occur. This requires that the separation of the planes, $H$ be greater than a value that depends on the applied stress. 


\section{FIGURE CAPTIONS (CONTINUED)}

Figure 4 - Further motion of the dislocation lines of Figure 3. The configuration at the center has been restored to its form in Figure 2, the original line on the lower plane is moving off to the right, and there is a new loop on the upper plane with one segment moving off to the left, and the other to the right.

Figure 5 - Cnritinuation of the motion of the dislocation lines in Figure 2 when the separation of the planes, $h$ is too small for the lines to pass over one another under the given applied stress. Two edge dislocation dipoles are left behind as the original line moves forward (portions being on two different planes).

Figure 6 - Experimental observations of shear bands forming from half-loops. The material is a lithium fluoride crystal. The half-loops were produced by lightly deforming the surface and then chemically polishing away most of the dislocations until only a few pairs of pits remained. Each pair designates the two ends of a half-loop emerging from the surface. Tension applied in the horizontal direction so that six half-loops were subjected to shear stress while a seventh was not. Strain-rate was about $10^{-5} / \mathrm{sec}$. (a) Set of half-loops at the surface. (b) same crystal surface after stress pulse was applied. Shadows of shear bands pass through five of the half-loops. The sixth one appears to have been blocked by the sub-grain boundary. (c) A light etch reveals a multitude of dislocation etch-pits in each of the shear bands. 
Figure 7 - Lateral growth of a shear band. The photograph shows the etched surface of a lithium fluoride crystal that was plastically deformed twice, and etched after each deformation. The width of the shear band was $W_{1}$ after the first deformation, and $W_{2}$ after the second deformation. Magnification, 300x. Note the relatively uniform density of etchpits (dislocation lines) within the bands, indicating that most of the motion as a band widens is at its edges (because its center has become strain-hardened).

Figure 8 - Stress-strain curve of Mg0 crystal at room temperature, and corresponding sketches of the shear band structure after various amounts of plastic strain. Note that macroscopic hardening begins at Point $C$ where the shear band has first filled the entire gauge length. Note the slight instability near Point $A$. For various similar specimens the slope, $\theta$ lay between $G / 200$ and $G / 300$; values comparable with Stage II hardening of face-centered-cubic metals. After Stokes, 1964 .

Figure 9 - Showing the large dependence of dislocation multiplication-rates on applied stress. The graph shows the linear density of dislocation loops after a stress pulse of fixed duration (variable stress) has been applied to a lithium fluoride crystal. The logarithm of the loop density is plotted vs. the reciprocal shear stress. Thus the multiplication-rate rule is: $\Lambda=\Lambda_{0} \exp (-S / \tau)$ where $S$ is the slope in Figure 9. 
Figure 10 - Schematic Pareto dipole distribution. $L$ is the dipole concentration $\left(\# / \mathrm{cm}^{2}\right) ; L_{0}$ is the concentration of the ones with the smallest moment, $h_{0}$; and $h$ is the moment (height) up to the limiting value, $h *$ which is determined by the applied stress. $h_{0} \approx b$.

Figure 11 - Calculated creep curve compared with experiment. The data are for a lithium fluoride crystal. The only adjustable parameter was the hardening coefficient. The shape of this curve also describes the shape of the edge of a shear band because the time derivative of the plastic strain is related to the space derivative through the continuity equation when the stress is constant. 
$-27-$

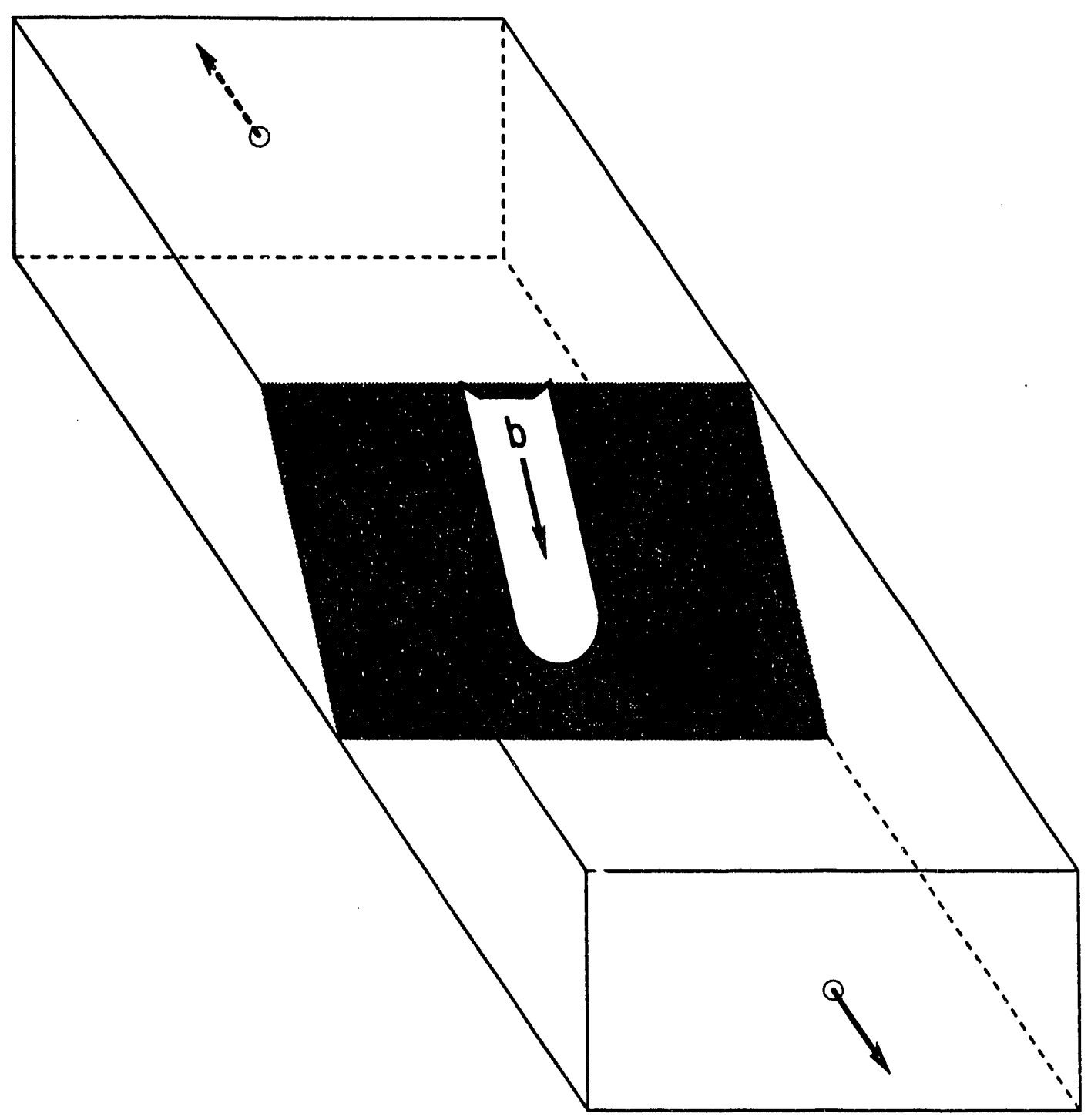

Figure 1 
$-28-$

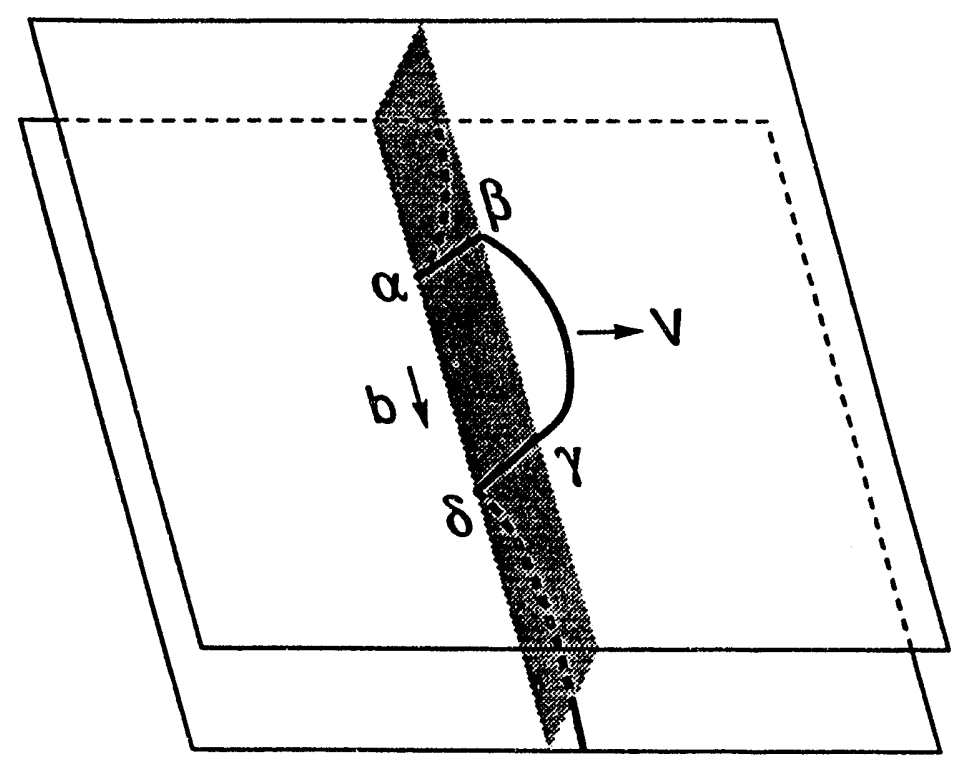

Figure 2 


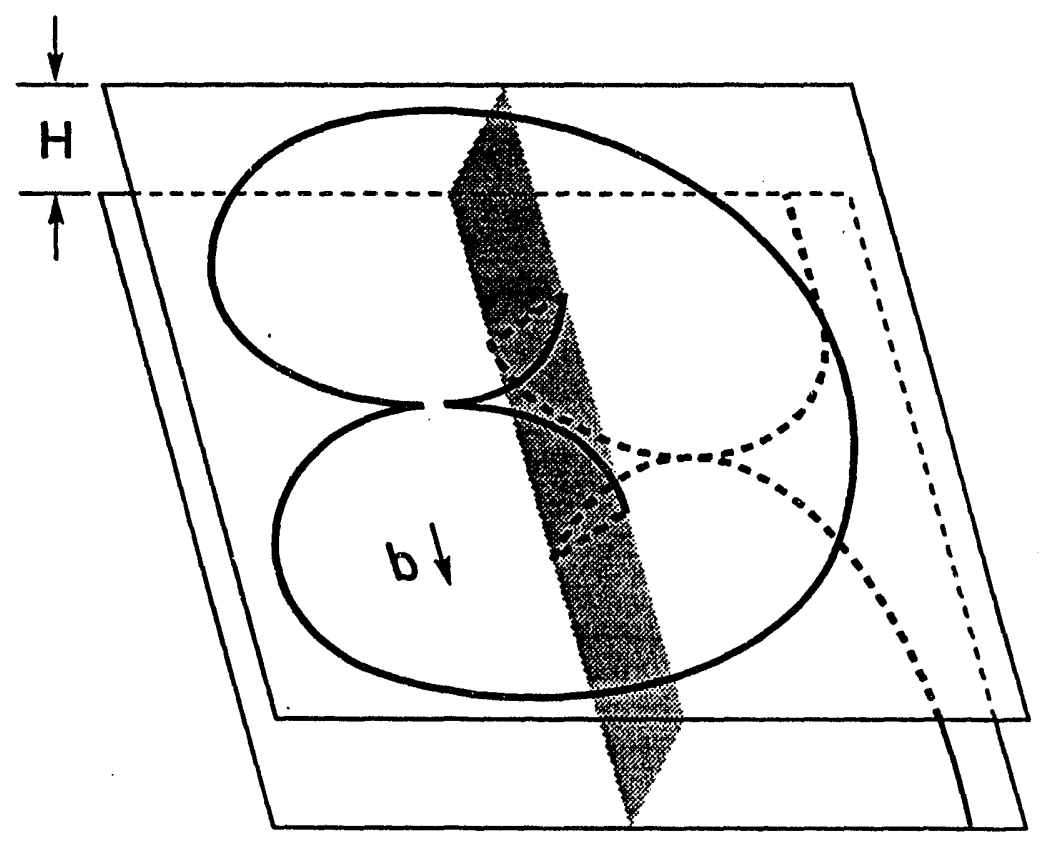

Figure 3 
$-30-$

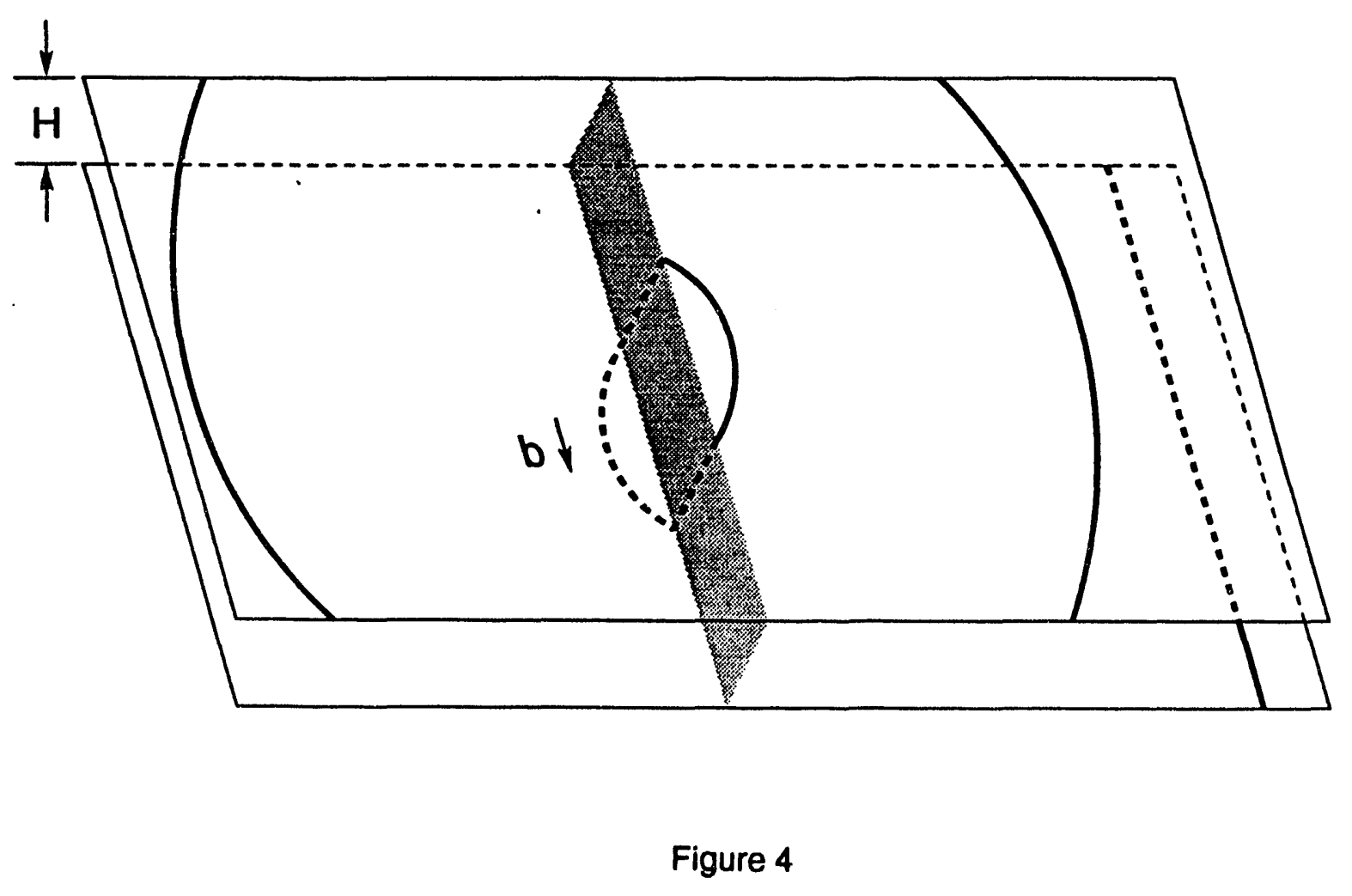




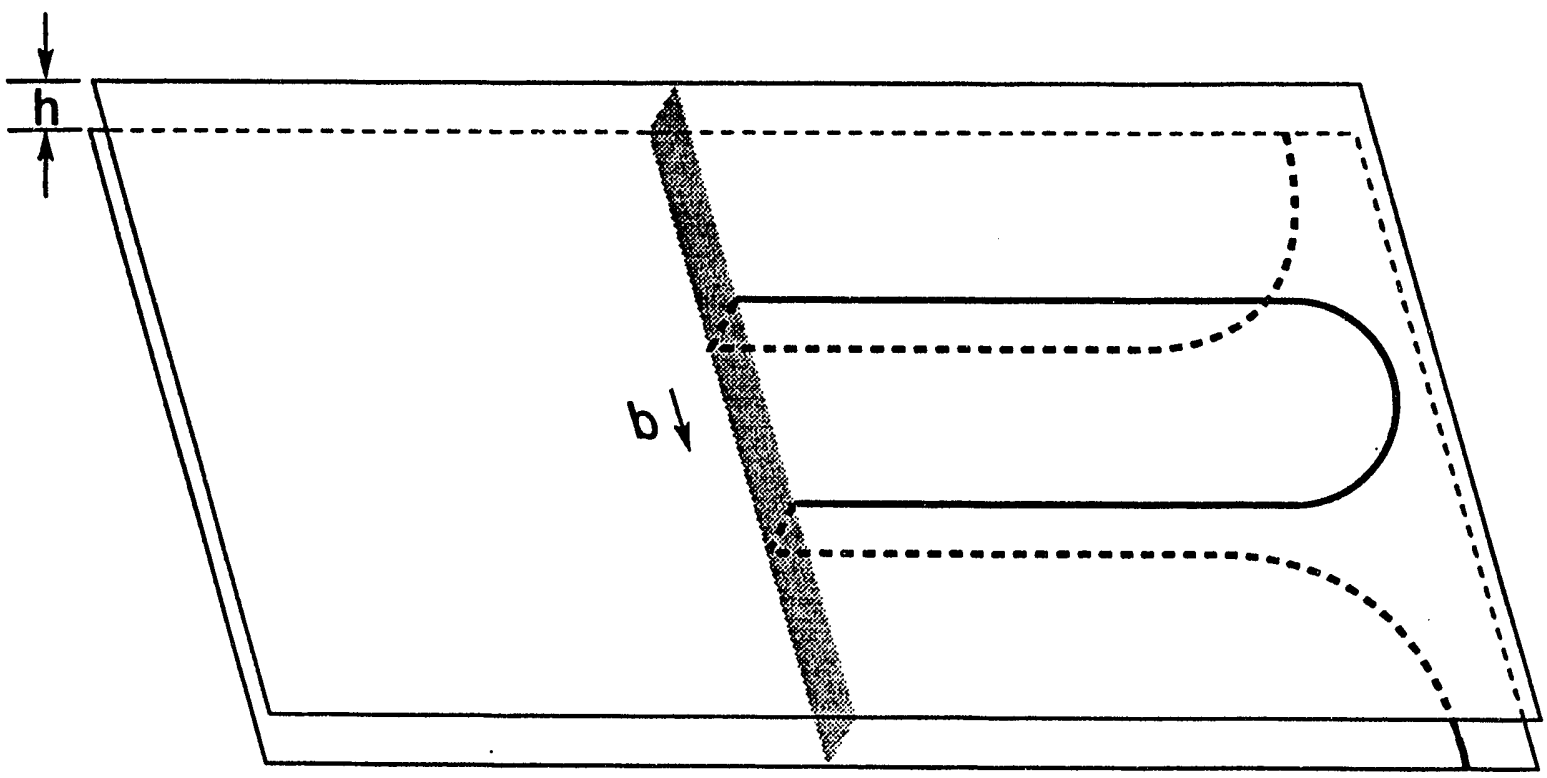

Figure 5

$\wedge$ 



Figure 6 


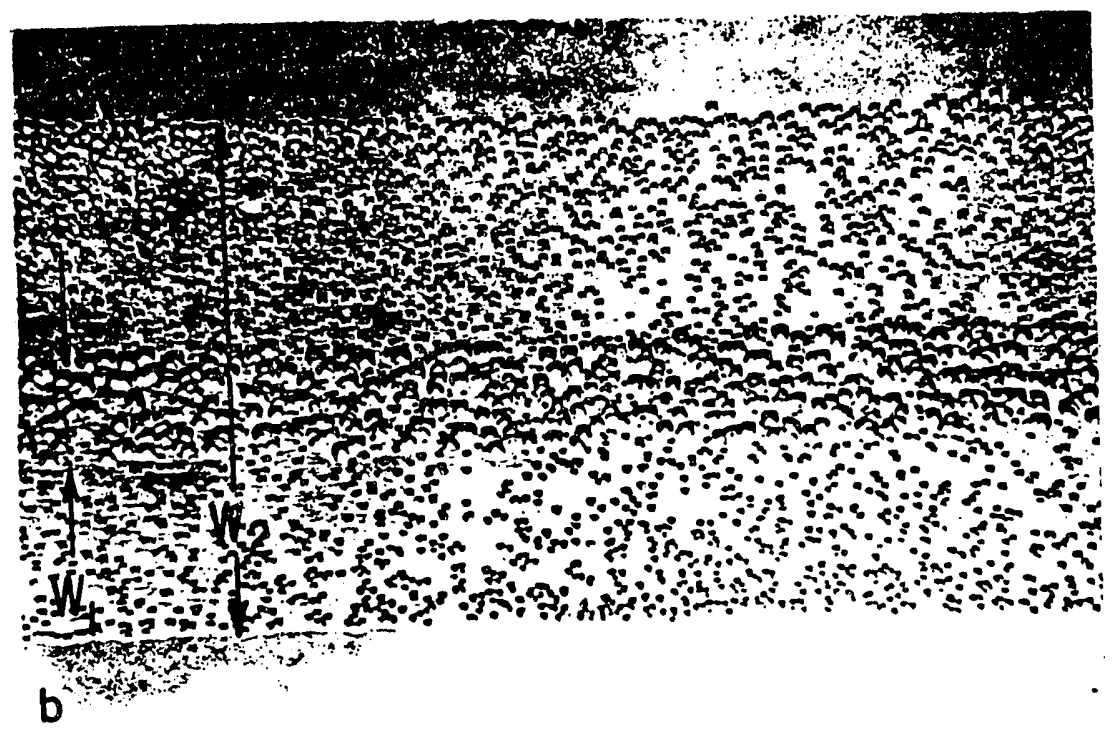

Figure 7 


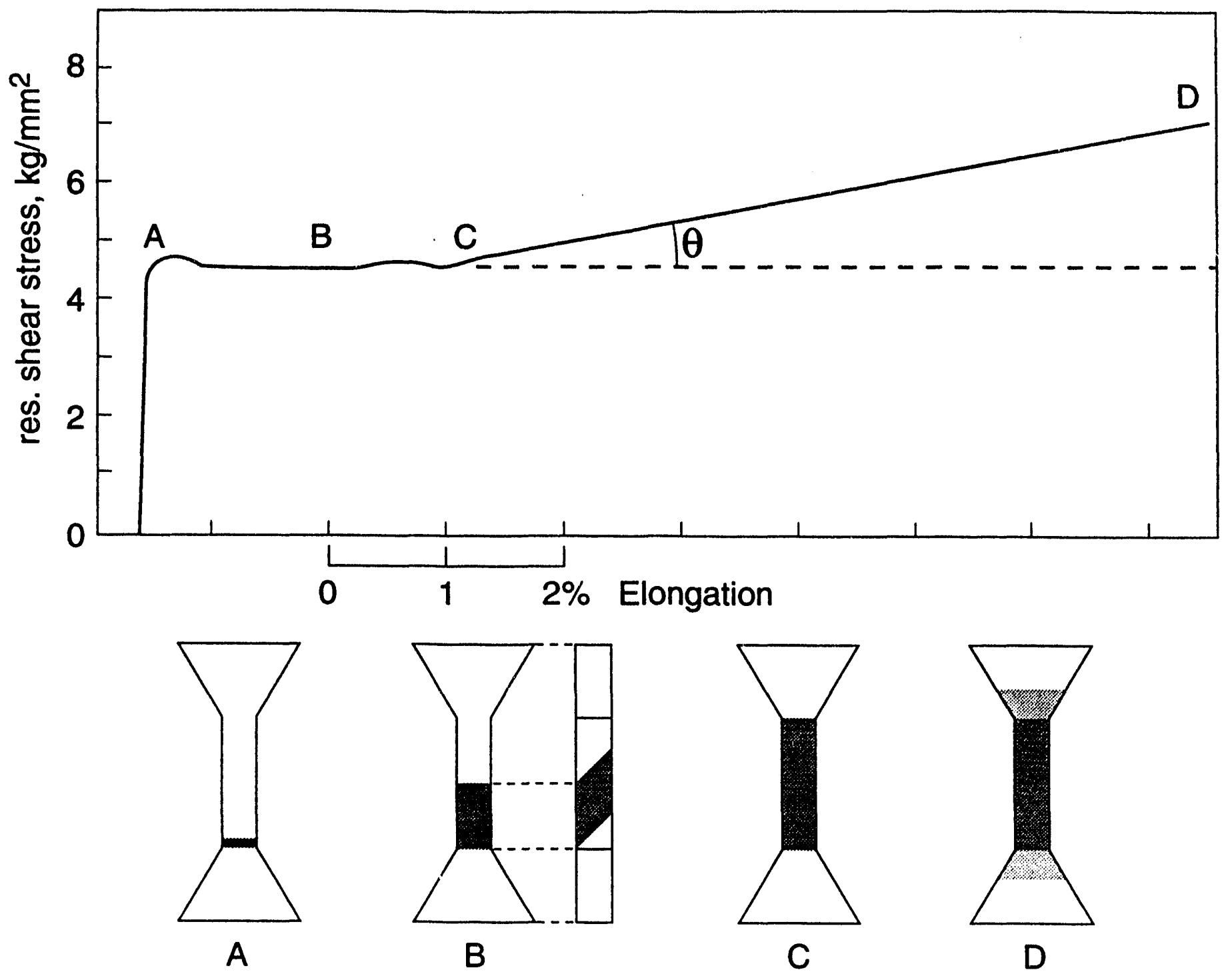

Figure 8 


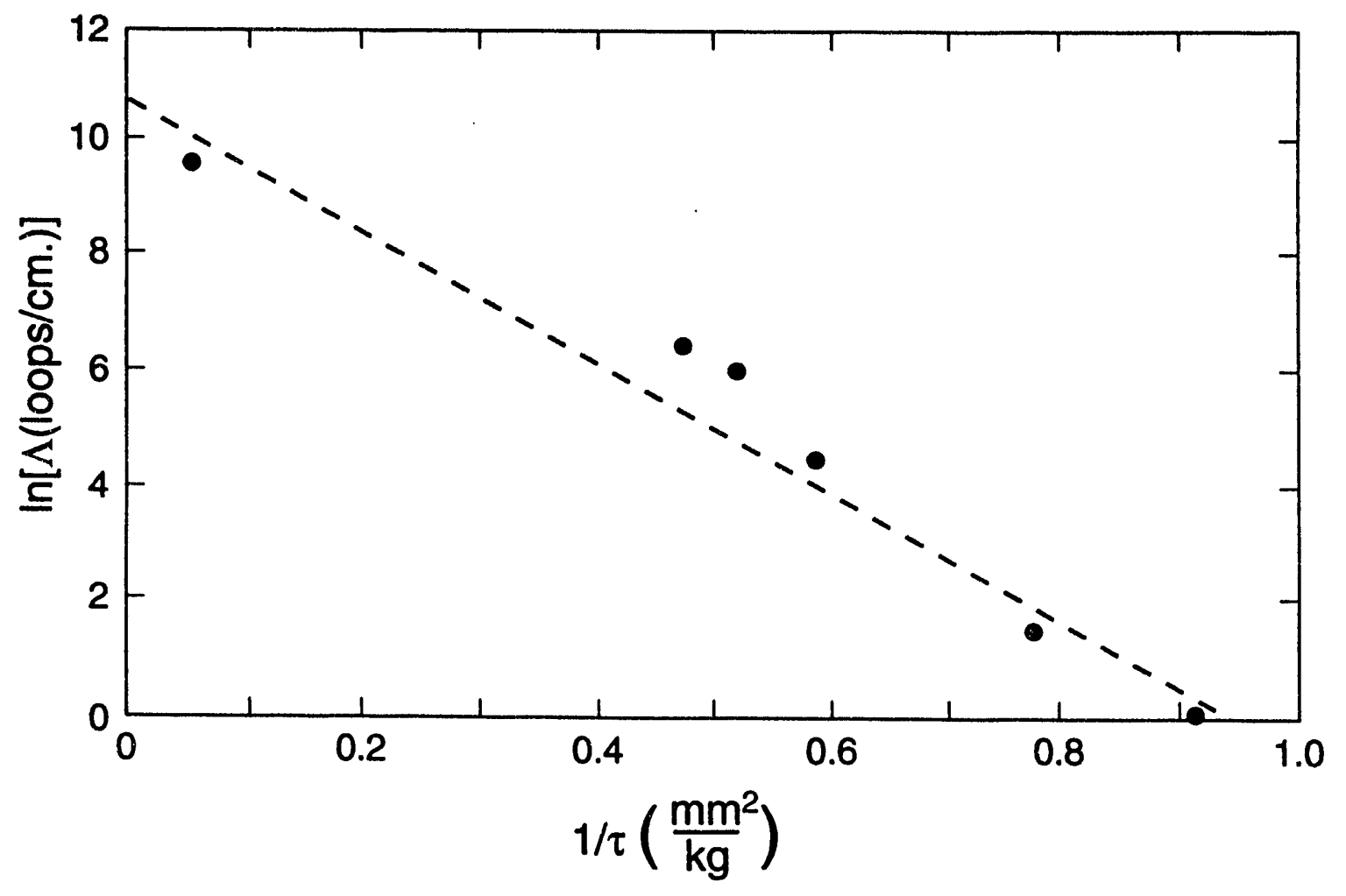

Figure 9 


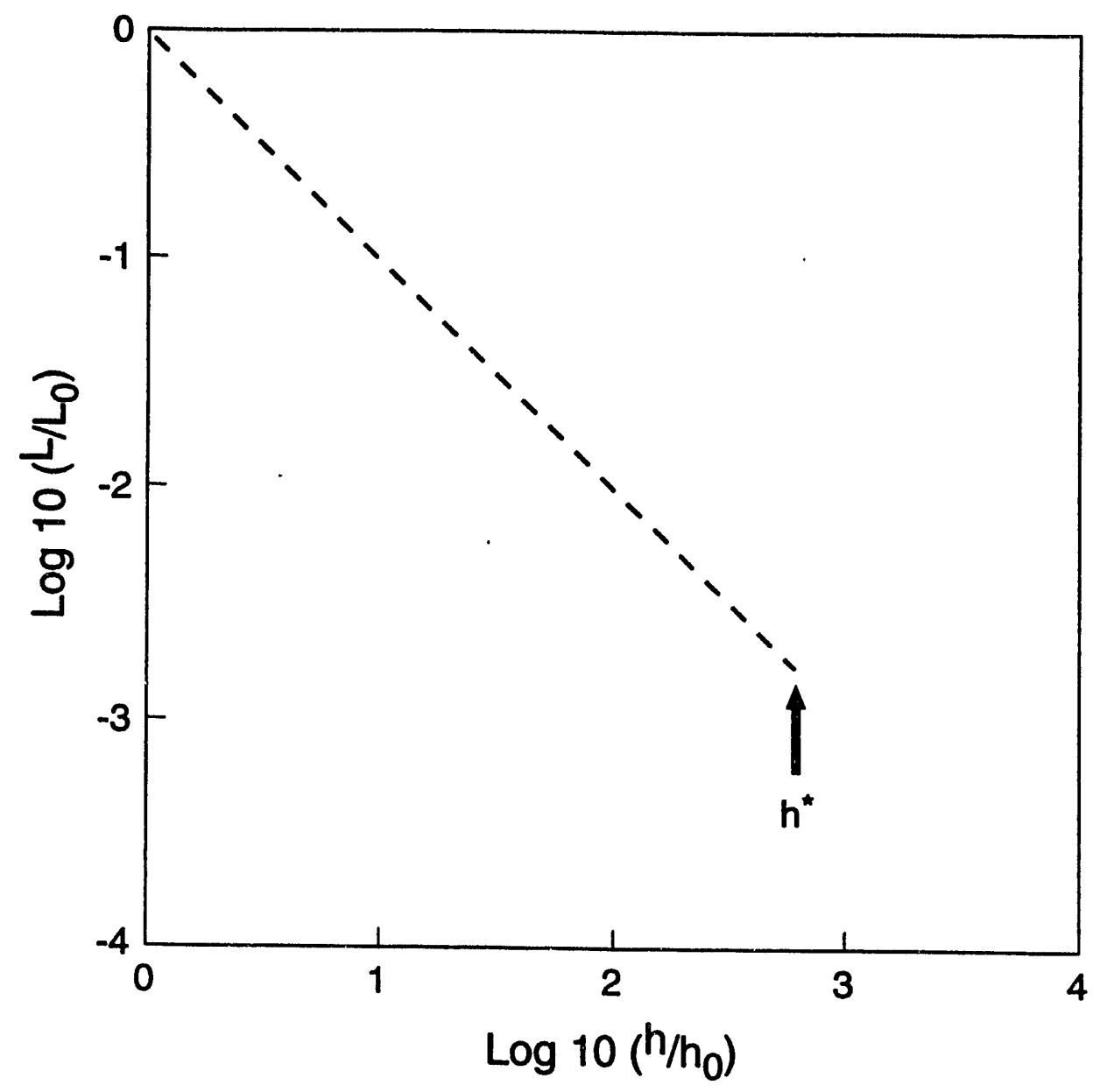

Figure 10 
$-37-$

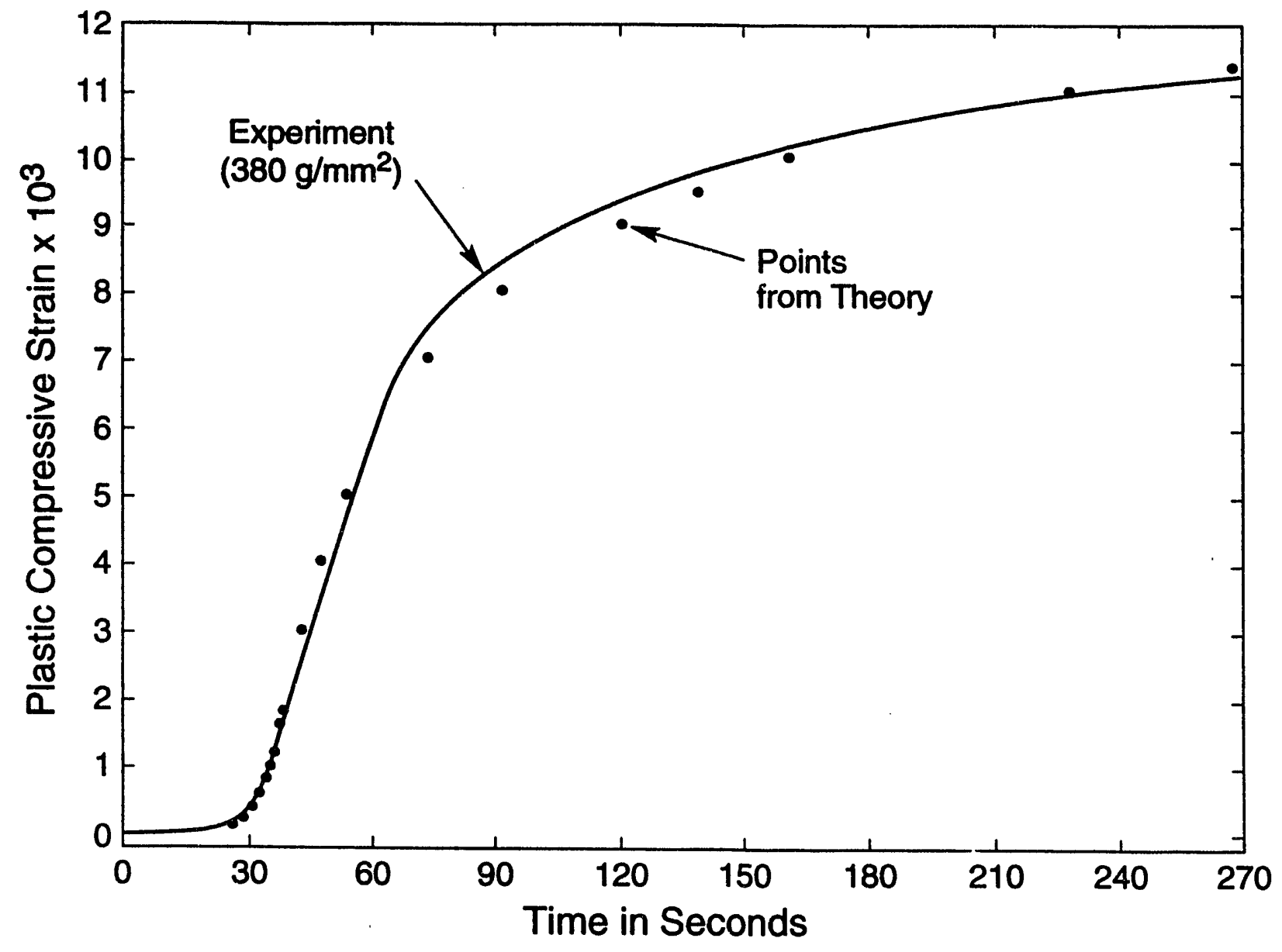

Figure 11 

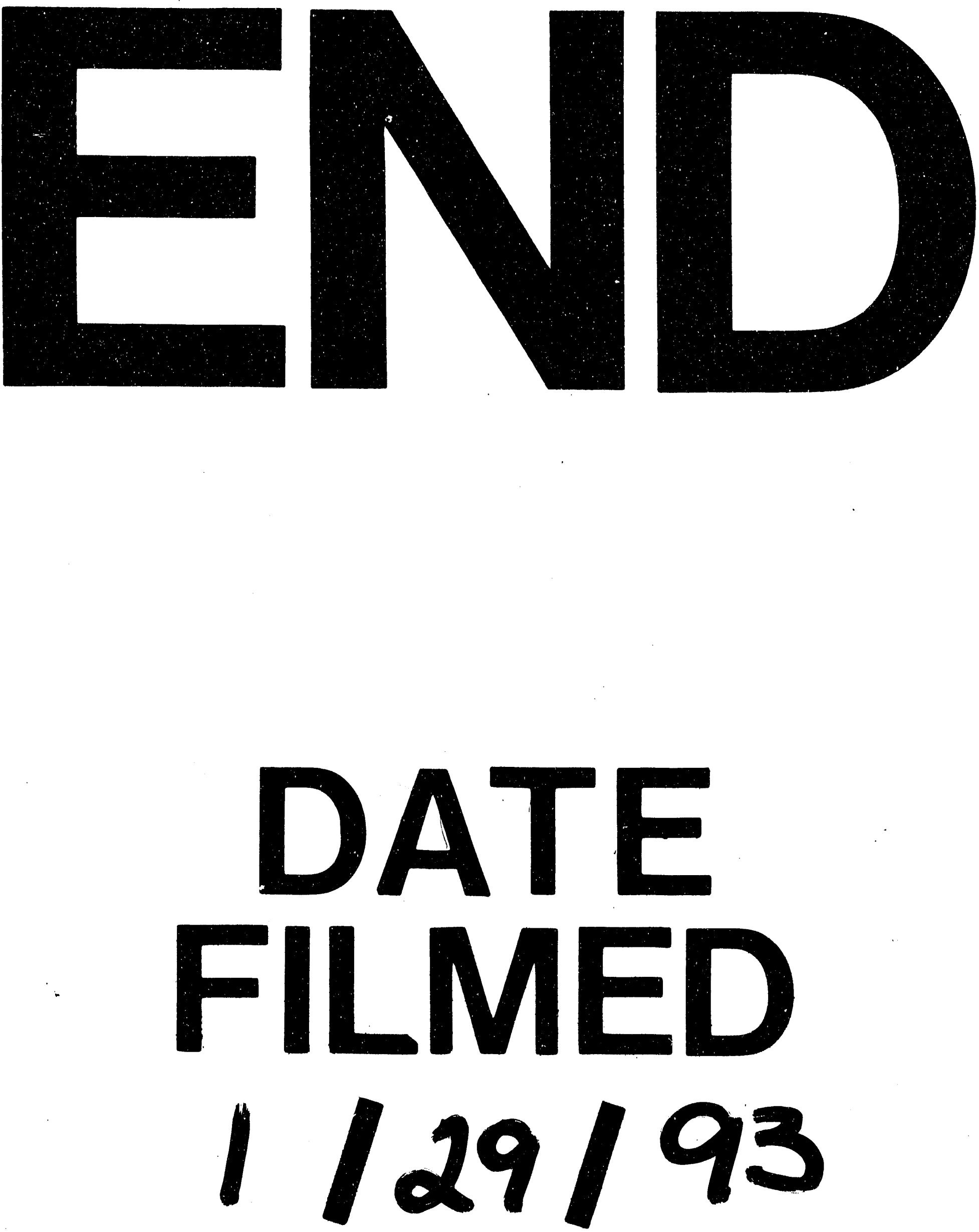
